\title{
Study on Distribution Optimal Drugs in Eradicating Ebola
}

\author{
Xinjun Li \\ School of Electric Power Engineering, North China Electric Power University, Baoding 071000, \\ China.
}

985061249@qq.com

Keywords: Graph theory method, Lingo, Allocation optimization, Simulation.

\begin{abstract}
The drug allocation model is set up to optimize the distribution of drugs after the outbreak. Considering that the severity of the epidemic areas are different and their economic level is low, we establish the network of service stations and the epidemic stricken areas. Then the dynamic drug distribution model is built, which consists of two stages. In the early stage, drug is shortage. For the effective control of the epidemic, minimizing daily new infections number as the goal, and distribute drugs according to the situation in the disaster areas. In the later stage, drug is enough, with the goal of minimum cost, employing the knowledge of graph theory, the distribution is given. Finally, we apply the model to a case, and the model's rationality is verified by the results.
\end{abstract}

\section{Introduction}

In the event of the Ebola virus outbreak, drug delivery is crucial for emergency demand response. Here, based on the spread of the Ebola disease and the extent of the disaster area, we study the optimal scheme for the situation with multiple distribution sites and disaster areas. First, according to the local transportation conditions and the topographic distribution of disaster area, the distribution location is determined. Then, given the main reasons for the outbreak in Africa: the low level of productivity and imperfect medical facilities, the supply of drugs are bound to affect the drug distribution scheme. Only when the speed of the drug production reaches a certain degree, can the demand of epidemic areas is met. So we have to consider the distribution in two stages, the former stage lacking drug and the stage with adequate drug.

\section{Build the model}

\subsection{Drug shortage period}

\section{- Analysis}

In the early time, the drug production ability is low, and the limited drug satisfy the demand. To effectively control the spread of the epidemic, we regard the epidemic area total prevalence in all the fewest number as the goal, optimizing drug distribution of the epidemic area.

- Assumptions

$>$ Assume that the area's drug demand is proportional to the local cure rate

$>$ The number of the diagnosed is equal to the incidence

- symbols and definitions

\begin{tabular}{cl}
\hline Symbol & definition \\
\hline$\Delta C_{t k}$ & the new onset of incidence number in the day i of the disaster $\mathrm{k}$ \\
$L_{t k}$ & the latency number in the day i of the disaster $\mathrm{k}$ \\
$C_{t k}$ & the latency number \\
$r_{k}$ & the ratio of total healers by the day $\mathrm{t}$ \\
$W_{t k}$ & the rug distribution of the disaster in the day $\mathrm{t}$ \\
$\mathrm{l}$ & the diary ratio that lurk become the incidence \\
$N_{t}$ & the total rug distribution in the day $\mathrm{t}$ \\
\hline
\end{tabular}

model

This is a resource optimization problem, at this stage, to more effectively control the whole 
epidemic situation, we regard the epidemic area total prevalence in all the fewest number as the goal.

Seeing the goal, every area has its rug distribution demand, which is proportional to the local cure rate. Based on the assumption, we find that the distribution is also proportional to the local cure rate. Among that, $\beta$ is a constant per day, and $\beta>0$.

Considering the rug is limited, but increase along with the time, assume the amount of the rug per day is $N_{t}$.

So, the optimization model is

\subsection{Drug sufficient period}

$$
\begin{gathered}
\text { s.t } \min \sum_{k=1}^{n} \Delta C_{t k}=l L_{t k}-r_{k} C_{t k} \\
\left\{\begin{array}{l}
W_{t k}=r_{k} \cdot \beta \\
\sum_{k=1}^{n} W_{t k} \leq N_{t} \\
\beta>0 \\
r_{k} \cdot \leq 30 \%
\end{array}\right.
\end{gathered}
$$

- analysis

In the later time, the drugs are availability. In view of the low local economic level, we regard the lowest transportation cost as the goal, using graph theory method for the service station to the area of drug the distribution plan.

- assumptions:

$>$ Assume that the locations of the distribution centers also are previously known.

$>$ There are enough vehicles to deliver the vaccine without loss.

- symbols and definitions

\begin{tabular}{cl}
\hline Symbol & definition \\
\hline$L_{i j}$ & the distance between the destination $\mathrm{i}$ to the disaster $\mathrm{j}$ \\
$Q_{j}$ & the drug demand of the disaster $\mathrm{j}$ \\
$\lambda$ & the unit price transporting unit medicine in unit distance \\
$X_{i j}$ & the drug quantity transport from the destination to the disaster $\mathrm{j}$ \\
$W_{i}$ & the drug quantity of the destination $\mathrm{j}$ \\
$\mathrm{Z}$ & the total transportation cost \\
\hline &
\end{tabular}

This is still a resource optimization problem, at this stage, drug is enough. Considering the actual local economy level is low, we aiming at maximum saving transportation cost.

The service station to the disaster area transportation demand for drugs sum equal to the area of drugs.

In real life, service stations' total number of export drugs of service station can't be more than the number of their drugs.

So, this optimization problem show as below:

$$
\begin{aligned}
\text { s.t } \quad \min Z=\sum_{i=1}^{m} \sum_{j=1}^{n} \lambda L_{i j} X_{i j} \\
\left\{\begin{array}{l}
\sum_{i=1}^{m} X_{i j}=Q_{j} \\
\sum_{j=1}^{n} X_{i j} \leq W_{i} \\
X_{i j} \geq 0
\end{array}\right.
\end{aligned}
$$




\section{Simulation of disease}

Now, there are five Ebola epidemic areas, two service station. When the disease outbreak, the total amount of drug of two service stations is 100, in which add 10 per day. The drug is enough at the $44^{\text {th }}$ day. In this period of time, assume that there is no person die of disease in this area. The population is constant.

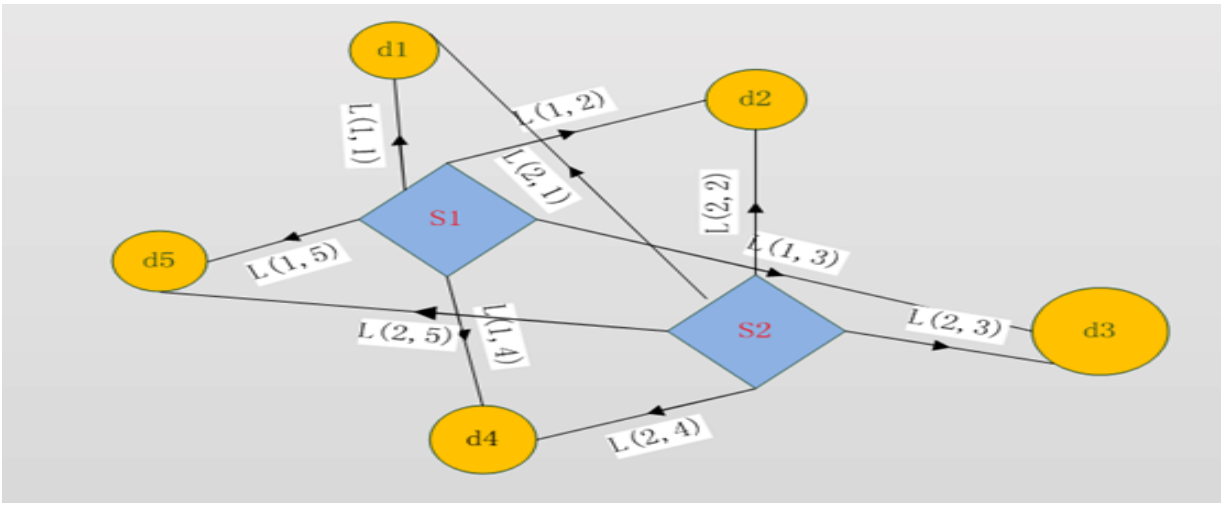

Fig. 1 The distribution of known service stations and the epidemic areas

The population of the five epidemic areas $\{\mathrm{d} 1, \mathrm{~d} 2, \mathrm{~d} 3, \mathrm{~d} 4, \mathrm{~d} 5\}$ are $\{1100,1200,1300$, $1400,1250\}$,When lack of drugs, the area of drugs to be delivered is proportional to the cure rate, the ratio is 600 .

According to the epidemic situation prediction, the number of drugs of $\{\mathrm{d} 1, \mathrm{~d} 2, \mathrm{~d} 3, \mathrm{~d} 4, \mathrm{~d} 5\}$ is $\{374,408,442,743,695\}$

The distance between service stations and epidemic areas show as below:

Table 1 The distance between service stations and epidemic areas

\begin{tabular}{|c|c|c|c|c|c|}
\hline$L_{i j}$ & d1 & $\mathrm{d} 2$ & d3 & $\mathrm{d} 4$ & d5 \\
\hline $\mathrm{S} 1$ & 10 & 15 & 40 & 16 & 9 \\
\hline $\mathrm{S} 2$ & 38 & 17 & 10 & 11 & 30 \\
\hline
\end{tabular}

According to the data, draw the change curves of the total incidence in the epidemic areas before and after optimal drug distribution.

Analysis:

We can know from the question, 1-43 days after the outbreak is the drug-lack period, drug is enough from the $44^{\text {th }}$ day on. Therefore, distribute drugs on the next day by the method of model in 2.1 and distribute drugs on the $45^{\text {th }}$ day by the method of model in 2.2. The distribution shows as below:

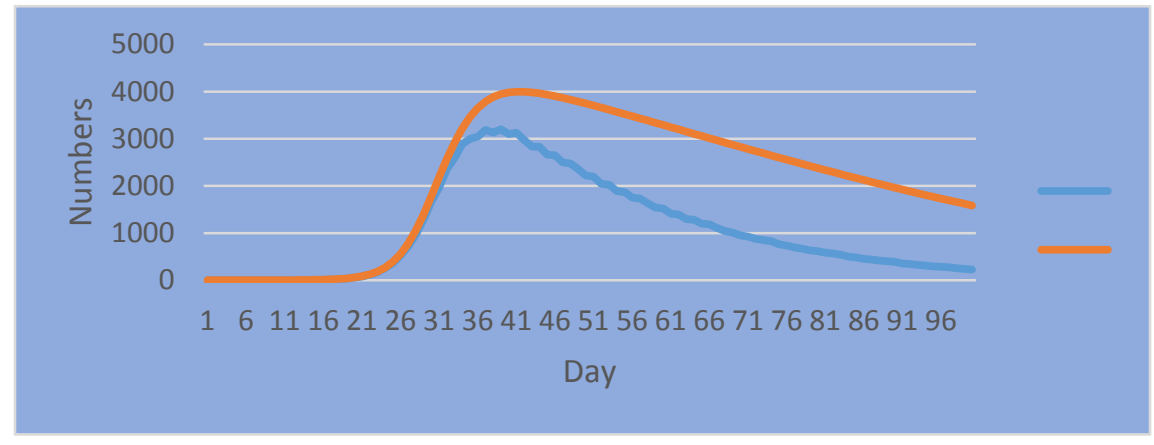

Fig .2 The curves of the total incidence before and after optimal drug distribution

To calculate the number of export drug to epidemic areas from each service station on $45^{\text {th }}$ day, 
show as below:

Table 2 the distribution on the $45^{\text {th }}$ day

\begin{tabular}{llllll}
\hline & $\mathrm{d} 1$ & $\mathrm{~d} 2$ & $\mathrm{~d} 3$ & $\mathrm{~d} 4$ & $\mathrm{~d} 5$ \\
\hline $\mathrm{S} 1$ & 374 & 408 & 0 & 107 & 442 \\
$\mathrm{~S} 2$ & 0 & 0 & 743 & 588 & 0 \\
\hline
\end{tabular}

The cost of transportation on this day $\mathrm{Z}=29448$, the export of service station S1 and S2 both are 1331, that is to say, the manufacture speed of each service station on $44^{\text {th }}$ day both are 1331 .

\section{Summary}

Considering the drug production and transportation, we divide the drug delivery system into two stages respectively and optimize it.

\section{References}

[1] http://wenku.baidu.com/view/cbfaec0103d8ce2f00662373.html

[2] http://www.guokr.com/article/439133/

[3] http://baike.sogou.com/v168387.htm

[4] http://en.wikipedia.org/wiki/Ebola_virus_disease

[5] http://www.doc88.com/p-0893982493524.html 